\title{
Effects of Physical Form of a Starter for Dairy Replacement Calves on Feed Intake and Performance
}

\author{
A. Bach, ${ }^{*} \dagger^{1}$ A. Giménez,‡ J. L. Juaristi, $\neq$ and J. Ahedoł \\ *Institució Catalana de Recerca i Estudis Avançats (ICREA), 08010 Barcelona, Spain \\ †Grup de Recerca en Nutrició, Maneig, i Benestar Animal, Unitat de Remugants, Institut de Recerca i Tecnologia Agroalimentàries (IRTA), \\ 08193 Bellaterra, Spain \\ ‡Rancho Las Nieves, 50550 Mallén, Spain
}

\begin{abstract}
One hundred six female Holstein calves [body weight $(\mathrm{BW})=41.5 \pm 0.37 \mathrm{~kg}$ and $11.2 \pm 0.3 \mathrm{~d}$ old $]$ were used to evaluate the effects of physical form of a starter on animal performance and starter intake. Calves were randomly allocated to 2 treatments consisting of either a multiparticle or a pelleted starter. Both starters had exactly the same ingredient and nutrient composition but differed in their physical form. Calves received 4 $\mathrm{L} / \mathrm{d}$ of the same milk replacer at a $150 \mathrm{~g} / \mathrm{kg}$ dilution rate in 2 offers of $2 \mathrm{~L}$ each until they consumed an average of $300 \mathrm{~g} / \mathrm{d}$ of starter (as fed) for 2 consecutive days; then the dilution rate was decreased to $120 \mathrm{~g} / \mathrm{kg}$ until the age of $49 \mathrm{~d}$ when milk replacer was limited to 1 daily dose of $2 \mathrm{~L}$ until $57 \mathrm{~d}$ of age. Calves were kept in individual hutches for at least 1 wk after weaning. Body weight was measured at the beginning of the study and at 49 and $64 \mathrm{~d}$ of age. The median perimeters for the multiparticle and pelleted starters were $0.61 \pm$ 0.016 and $2.71 \pm 0.082 \mathrm{~cm}$, respectively. Overall starter consumption was greater in calves receiving the multiparticle starter $(944.8 \pm 30.01 \mathrm{~g} / \mathrm{d})$ than in those receiving the pelleted starter $(863.9 \pm 32.04 \mathrm{~g} / \mathrm{d})$. There were no differences in the total milk replacer intake between the 2 treatments. Calf BW when leaving the individual hutches at the end of the study was similar between both treatments. Consequently, feed conversion efficiency was greater in calves consuming the pelleted than the multiparticle starter up to $64 \mathrm{~d}$ of age, mainly due to the greater conversion efficiency obtained with the pelleted than with the multiparticle starter after the preweaning period. It is concluded that pelleted starters may result in lower dry feed consumption compared with multiparticle starters, but because final BW was similar in both treatments, feed efficiency of calves consuming pelleted starters may be greater than that
\end{abstract}

Received November 15, 2006.

Accepted February 5, 2007.

${ }^{1}$ Corresponding author: alex.bach@irta.es of calves consuming multiparticle starters. Therefore, when feeding a starter with similar nutrient composition to the one used in this study, there seems to be an economic advantage associated with feeding the starter in a pelleted form compared with a multiparticle form. Key words: calf, particle size, average daily gain

\section{INTRODUCTION}

In current production systems, optimizing calf growth, especially at early stages of life when feed conversion efficiency is greatest (Kertz et al., 1998), is a key objective for any profitable heifer-raising operation. It is commonly believed that texturized or coarse starters lead to better performance than finely ground starters (Warner et al., 1973; Warner, 1991). However, the evidence in the literature that coarse or texturized starters should perform better than finely ground or pelleted starters is very limited. Warner et al. (1973) reported that calves fed a starter in mash form had greater intakes and daily weight gains than calves fed a starter in pelleted form. However, Owen and Larson (1986) compared the intake of calves fed a meal or a pelleted starter and did not report differences in either starter intake or calf performance. A more recent study (Franklin et al., 2003) concluded that a texturized starter resulted in better performance than a pelleted or a ground starter (with no differences between these 2). However, the starters were not exactly the same, because the ingredients and their proportions were different in each of the 3 physical forms of the starters were different. Furthermore, the weaning moment was also different between treatments.

The objective of this study was to evaluate the effects of the physical form a starter on feed intake and growth of dairy replacement calves.

\section{MATERIALS AND METHODS}

One hundred six female Holstein calves with an initial age of $11.2 \pm 0.30 \mathrm{~d}$ and an initial BW of $41.5 \pm 0.37$ $\mathrm{kg}$ were kept in individual hutches $(1.07 \times 1.60 \mathrm{~m})$ in 
Table 1. Ingredient and nutrient composition of the experimental starter $^{1}$

\begin{tabular}{lc}
\hline Item & \\
\hline Ingredients & \\
Wheat middlings & 29.6 \\
Corn, cracked & 20.0 \\
Oats, whole & 14.6 \\
Soybean hulls & 8.4 \\
Soybean meal & 8.0 \\
Corn gluten meal & 7.3 \\
Carob & 5.2 \\
Cookie's waste & 2.2 \\
Calcium carbonate & 5.8 \\
Sodium chloride & 0.5 \\
Vitamin and trace mineral premix ${ }^{3}$ & 0.4 \\
Nutrients & 89.0 \\
DM, \% & 21.9 \\
CP, \% of DM & 4.1 \\
Ether extract, \% of DM & 28.8 \\
NDF, \% of DM & 2.89 \\
ME, Mcal/kg & \\
\hline
\end{tabular}

${ }^{1}$ Units are percentage of DM unless otherwise noted.

${ }^{2}$ Cookie's waste is a by-product of the cookie industry.

${ }^{3}$ Premix contained (mg/kg): vitamin A, 4,200; vitamin D, 1,575; vitamin $\mathrm{E}, 42 ; \mathrm{Fe}, 15 ; \mathrm{Zn}, 35 ; \mathrm{Cu}, 11.6 ; \mathrm{Mn}, 16.3$; Co, 0.13; I, 0.46; and Se, 0.35 .

${ }^{4}$ Calculated following the NRC (2001).

the facilities of Rancho Las Nieves (Malln, Spain). The hutches were bedded every $3 \mathrm{~d}$ with chopped straw. The calves arrived at Rancho Las Nieves from 46 Spanish farms during July 2006. Upon arrival, calves were checked for health problems and randomly allocated to either a multiparticle starter or a pelleted starter. Both starters had exactly the same ingredient and nutrient composition (Table 1) but differed in their physical form; both contained whole oats, cracked corn, and cracked carob. The rest of ingredients were ground in the multiparticle starter, whereas in the pelleted starter all the ingredients were ground through a 2$\mathrm{mm}$ mesh and pelleted to a length of $15 \mathrm{~mm}$ and a diameter of $3 \mathrm{~mm}$.

Calves had ad libitum access to water and starter and received $4 \mathrm{~L} / \mathrm{d}$ of the same milk replacer $(25 \% \mathrm{CP}$ and $19.2 \%$ fat on a DM basis) at a $150 \mathrm{~g} / \mathrm{kg}$ dilution rate in 2 offers of $2 \mathrm{~L}$ each from the beginning of study until they consumed an average of $300 \mathrm{~g} / \mathrm{d}$ of starter (as fed) for 2 consecutive days. Then, the dilution rate was decreased to $120 \mathrm{~g} / \mathrm{kg}$ until the age of $49 \mathrm{~d}$, at which time milk replacer was decreased to 1 daily dose of 2 $\mathrm{L}$ at the same dilution rate from 49 to $57 \mathrm{~d}$ of age with the aim of stimulating the consumption of starter and easing the weaning process. The milk replacer was offered using a 2-L bottle with a nipple. Calves were kept in individual hutches for at least $1 \mathrm{wk}$ after weaning. Calves were not offered any roughage (although it is likely that they consumed some straw from bedding). Individual intakes of milk replacer were recorded daily, and consumption of starter was also recorded daily by measuring the amount of starter offered and the refusals at the following day. Orts were pooled weekly and DM determined on weekly pooled samples. Calves were weighed at the beginning of the study and at $49 \pm 0.5$ and $64 \pm 0.2 \mathrm{~d}$ of age using an electronic scale (Allflex, Madrid, Spain).

Animals were vaccinated against Clostridium perfringens (types B, C, and D), Clostridium septicum, Clostridium novyi, Clostridium tetani, Clostridium sordellii, and Clostridium chauvoei with Miloxan (Merial, Lyon, France), and bovine respiratory syncytial virus, parainfluenza-3 virus, and Pasteurella haemolytica with Bovipast RSP (Intervet, Boxmeer, Holland) at 23 $\mathrm{d}$ of age and were revaccinated $3 \mathrm{wk}$ later.

Samples of milk replacer and starter were analyzed for $\mathrm{DM}\left(24 \mathrm{~h}\right.$ at $\left.103^{\circ} \mathrm{C}\right)$ and ash $\left(4 \mathrm{~h}\right.$ at $\left.550^{\circ} \mathrm{C}\right)$. Nitrogen content was determined using the AOAC (1990) method (988.05) adapted for an automatic Kjeldahl distillation unit (Kjeltec Auto 1030 Analyzer, Tecator, Höganäs, Sweden) using $\mathrm{CuSO}_{4}$ and Se as catalyst instead of $\mathrm{CuSO}_{4}$ and $\mathrm{TiO}_{2}$. Ether extract was analyzed using the AOAC (1990) method (920.39) using petroleum ether for distillation instead of diethyl ether. Neutral detergent fiber was assayed with heat-stable amylase and expressed including residual ash; ADF was also expressed including residual ash (Van Soest et al., 1991).

Instead of evaluating the particle size of the starters using different meshes, which would result in discrete proportions of particles at each mesh size, we opted for assessing the distribution of particle size in a way that allowed construction of a complete histogram of particle sizes of each starter. To accomplish this objective, the particle size distribution of the starters was determined using digital imaging by placing $50 \mathrm{~g}$ of starter on a smooth, plastic-coated black cardboard $(40 \times 40 \mathrm{~cm})$ with 2 scale bars of $5 \mathrm{~cm}$ each that were later used as a reference to convert pixels into centimeters by a computer measuring program. The sample particles were separated as much as possible and distributed across the cardboard surface by hand. Then, a digital picture with a resolution of $3,038 \times 2,012$ pixels and 16.7 million colors (about 5.9 megabytes) was taken (Nikon D70, Japan), framing the entire cardboard perpendicularly at an approximate distance of $60 \mathrm{~cm}$. Afterwards, the digital picture (raw format) was transferred to a personal computer and processed with a digital measuring program (Carnoy 2.1 for MacOSX, 2001). The digital measuring software determined the perimeter of each identified particle in the image and a complete perimeter distribution was constructed for each starter. 


\section{Calculations and Statistical Analysis}

Feed efficiency was calculated as the ratio of total BW gain and total DMI and was expressed as a percentage after multiplying by 100 .

All data referring to animal performance followed a normal distribution except for starter DMI that was logtransformed before analysis. Intake data were analyzed using a mixed-effects model with calf and farm origin as random effects, and type of starter, age, and the interaction between type of starter and age as fixed effects. Age entered the model as a repeated measure using a compound symmetry variance-covariance structure. All statistical analyses were performed with SAS (Version 9; SAS Inst. Inc., Cary, NC).

Differences in initial and final BW between the 2 treatments were assessed as a 1-way ANOVA. The effects of starter physical form on ADG as well as BW at preweaning time and at the age of $64 \mathrm{~d}$ were determined using a mixed-effects model with calf origin as a random effect and treatment and growth stage (preweaning or the end of the study) and their interaction as fixed effects. The effects of treatment on age at preweaning and weaning times, as well as on total milk replacer consumption, and total number of days consuming a milk replacer at a $15 \%$ dilution rate were assessed using an ANOVA with treatment as a fixed effect and calf origin as a random effect.

\section{RESULTS AND DISCUSSION}

The particle perimeter distributions of the 2 starters used in this study are depicted in Figure 1 . The median (the distribution was not normal) perimeter for the multiparticle starter was $0.61 \pm 0.016 \mathrm{~cm}$, whereas the median perimeter for the pelleted starter was $2.71 \pm$ $0.082 \mathrm{~cm}$. The multiparticle starter had only $7.8 \%$ of the particles with a perimeter greater than $2 \mathrm{~cm}$ and the distribution of perimeters was skewed to the left (due to the fact that the majority of the ingredients in the starter were ground), whereas the pelleted starter had more than $81 \%$ of the particles with a perimeter greater than $2 \mathrm{~cm}$ and the distribution of the perimeters was close to a normal distribution (because all the ingredients were ground and then pelleted).

Calves were preweaned (milk replacer was decreased to 1 daily dose) and weaned at the same age in both treatments ( $49.2 \pm 0.29$ and $57.5 \pm 0.28 \mathrm{~d}$, respectively), but because calves on the pelleted starter tended $(P=$ 0.07 ) to enter the study at an older age (on average, $1.7 \mathrm{~d}$ older), they received milk replacer for a numerically $(P=0.69)$ shorter number of days than the calves consuming the multiparticle starter. On the other hand, calves receiving the multiparticle starter tended $(P=$ 0.06 ) to consume the milk replacer at $15.5 \%$ dilution rate for a shorter period of time than calves receiving the pelleted starter $(23.3 \pm 1.27 \mathrm{vs} .25 .1 \pm 1.03 \mathrm{~d}$, respectively) because calves on the multiparticle starter consumed the target amount of starter $(300 \mathrm{~g} / \mathrm{d})$ to reduce the dilution rate of the milk replacer at an earlier $(P<$ 0.05 ) age (Figure 2 ). Therefore, calves on the multiparticle starter consumed milk replacer at the $12.5 \%$ dilution rate for longer $(P<0.01)$ periods than calves on the pelleted starter $(15.6 \pm 0.71$ vs. $12.1 \pm 0.60 \mathrm{~d}$, respectively). As a result, there were no differences in the total milk replacer intake between the 2 treatments (Table 2). In preparation for weaning, calves of both treatments spent the same amount of time $(8.2 \pm 0.31$ d) receiving a single dose of $2 \mathrm{~L} / \mathrm{d}$ of milk replacer at $12.5 \%$ dilution rate.

As expected, starter intake increased $(P<0.01)$ with age (Figure 2). The physical form of the starter had an effect on overall starter consumption. Calves receiving the pelleted starter consumed less $(P<0.01)$ starter $(863.9 \pm 32.04 \mathrm{~g} / \mathrm{d})$ than those receiving the multiparticle starter $(944.8 \pm 30.01 \mathrm{~g} / \mathrm{d})$. Other studies have also reported lesser consumptions of starters in pelleted than in mash form (Warner et al., 1973). However, in the current study, starter consumption was similar between the 2 treatments from the beginning of the study until the preweaning time ( $49 \mathrm{~d}$ ), but it was significantly $(P<0.01)$ lesser in calves receiving the pelleted starter than in those receiving the multiparticle starter thereafter (Figure 2 and Table 2).

Animals consuming the pellet starter tended $(P=$ $0.07)$ to enter the study at an older age than those on the multiparticle starter (Table 1), resulting in a numerical difference in BW of $1.31 \mathrm{~kg}$ between treatments at the beginning of the study. Nevertheless, the final BW when calves were leaving the individual hutches was similar in both treatments (with a numerical difference of $0.85 \mathrm{~kg}$ in favor of calves on the multiparticle starter). During the preweaning period (from 12 until $49 \mathrm{~d}$ ) both treatments sustained the same ADG, but calves on multiparticle starter grew numerically faster $(P=0.12)$ than calves consuming the pelleted starter (Table 2) once milk replacer was decreased to $2 \mathrm{~L} / \mathrm{d}$ (at $49 \mathrm{~d}$ of age) and later removed at $57 \mathrm{~d}$ of age. This greater ADG obtained with the multiparticle compared with the pelleted starter after the milk replacer was reduced and removed could be linked to the greater $(P<0.01)$ starter consumption of multiparticle than pelleted starter during this period.

Feed conversion efficiency $($ ADG/DMI $\times 100)$ was greater in calves consuming the pelleted starter than in those consuming the multiparticle starter. This difference in feed conversion efficiency was mainly due to a significantly greater $(P<0.01)$ efficiency in calves consuming the pelleted starter than in those on the 


\section{a) Multiparticle starter}

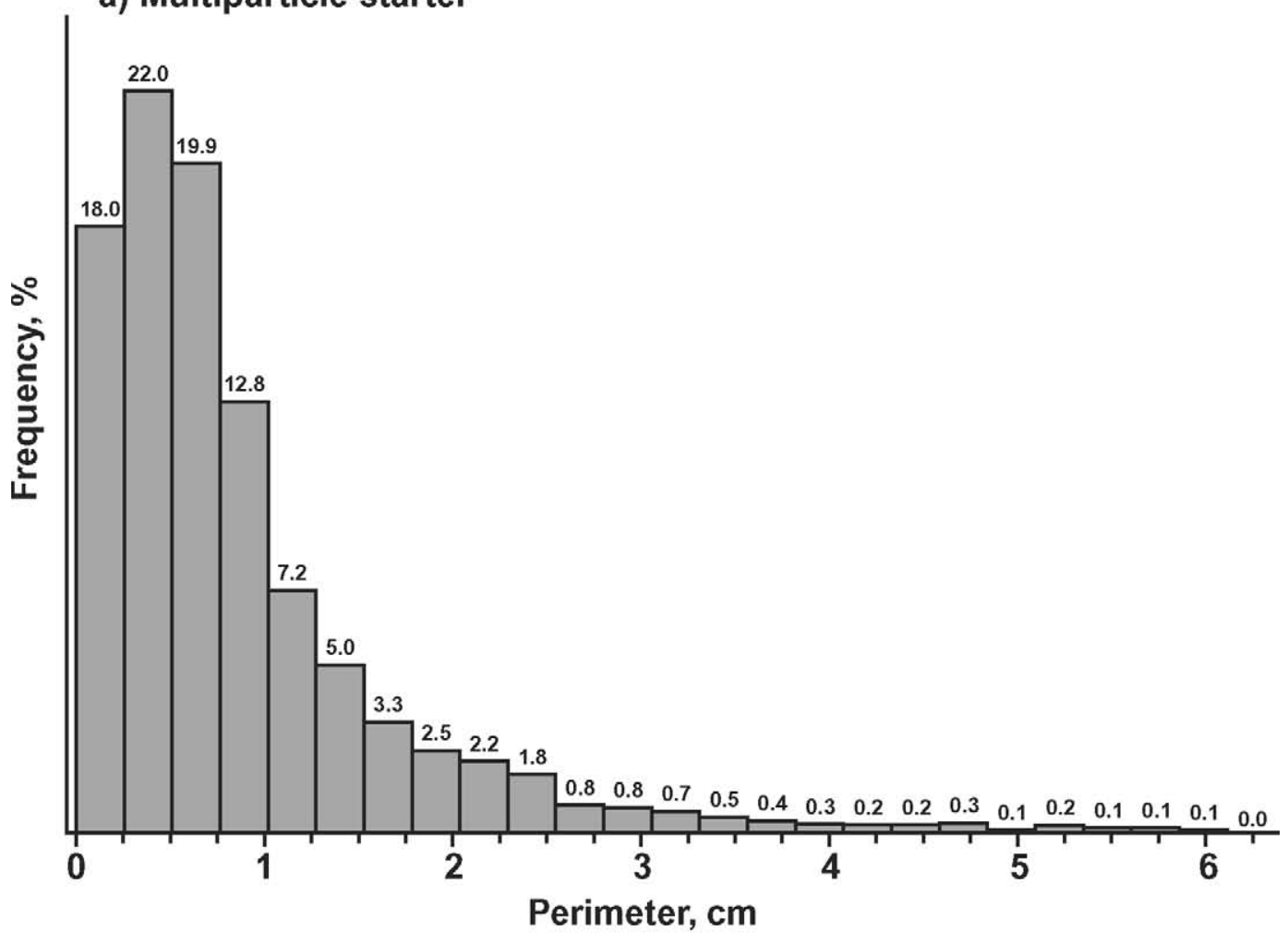

b) Pelleted starter

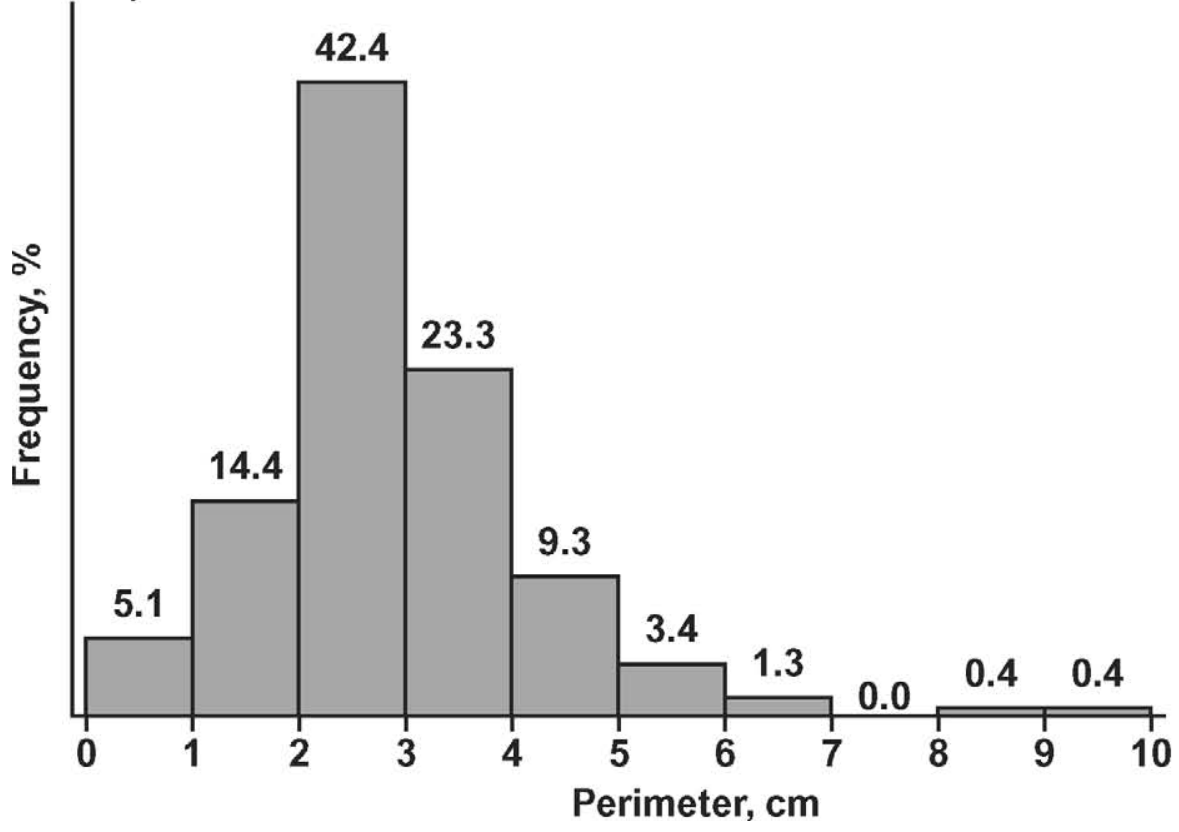

Figure 1. Particle perimeter distribution of the 2 experimental starters: a) multiparticle starter, b) pelleted starter.

multiparticle starter after the preweaning period (Table 2). Warner et al. (1973) concluded that starters in mash form with more than $50 \%$ of the particles greater than $1.2 \mathrm{~mm}$ were recommended because of an overall greater ADG of $30 \mathrm{~g} / \mathrm{d}$ when calves received such a starter compared with a starter in pelleted form. However, in that study the total amount of dry feed consumed by the calves receiving the starter in mash form 


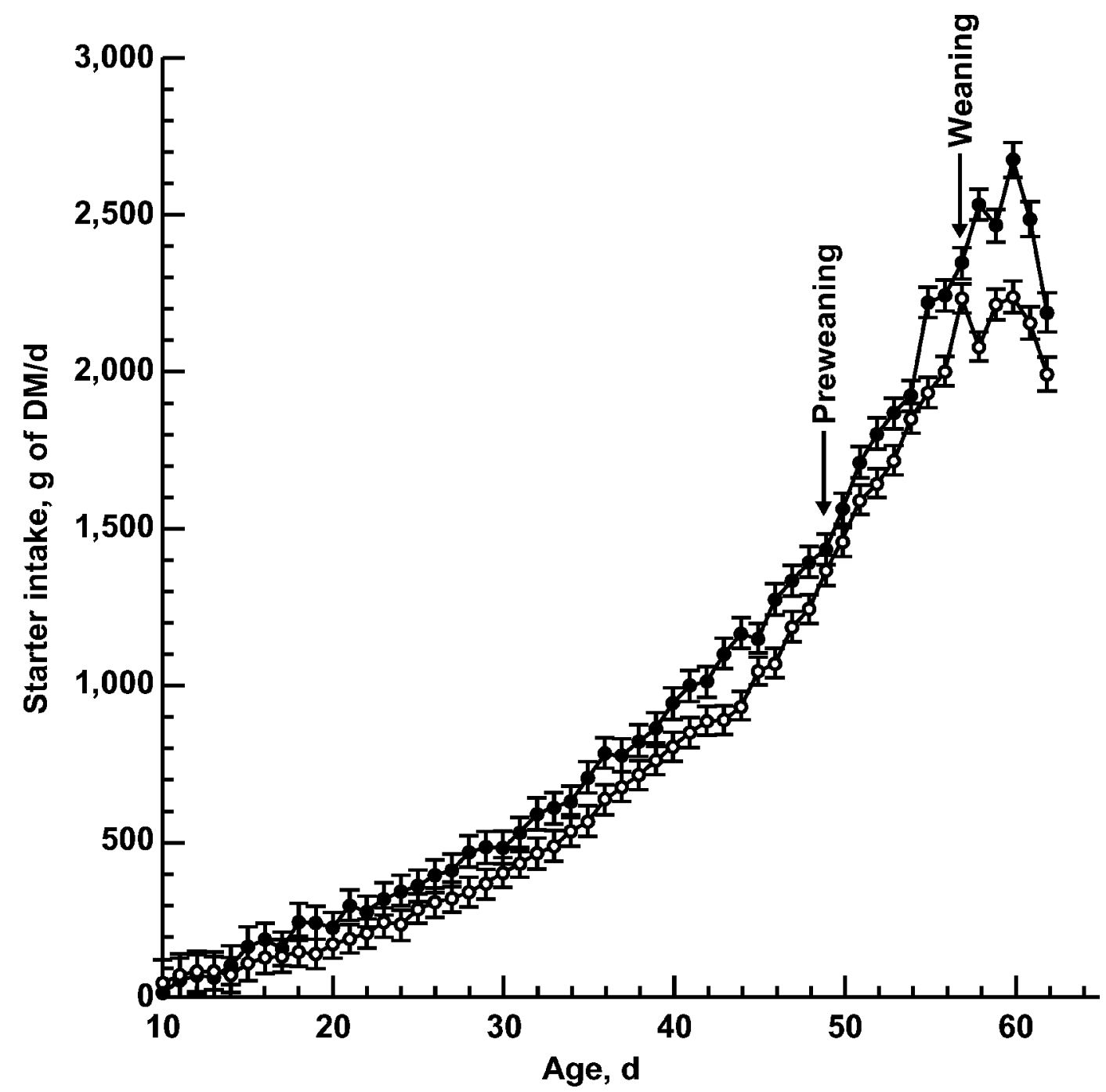

Figure 2. Effect of age and physical form of multiparticle $(\bullet)$ and pelleted $(\bigcirc)$ starter on intake of replacement calves.

was almost $16 \mathrm{~kg}$ less, which would suggest that calves receiving a pelleted starter would have greater feed efficiencies than calves consuming a starter in mash or multiparticle form, as occurred in the current study. In a more recent study, Franklin et al. (2003) also reported overall numerically (statistical analysis was not available) greater feed efficiencies in calves consuming a pelleted starter (65\%) than calves consuming a ground starter $(62 \%)$. However, in another study (Coverdale et al., 2004) there were no differences in performance, DMI, and feed efficiencies when comparing 2 multiparticle starters with a combination of pellets and either intact rolled corn and oats or ground corn and ground oats. In any case, an increase in feed conversion efficiency such as that observed in the current study coupled with no differences in calf final BW at $64 \mathrm{~d}$ of age would unequivocally lead to an economical advantage of feeding the pelleted vs. the multiparticle starter, not only because the same growth can be achieved using a lesser amount of starter, but also because a pelleted starter usually has a greater density, and thus the transportation costs from the production site to the farm could also be lesser than those of a multiparticle starter.

\section{CONCLUSIONS}

It is concluded that pelleted starters may result in lesser dry feed consumption compared with multiparticle starters, especially when the milk replacer is removed. This lower consumption can lead to lesser ADG between the time around weaning and about 2 mo of age. The combination of lesser dry feed intake and similar final BW implies that the feed efficiency of calves 
Table 2. Effect of physical form of the starter on calf performance and starter intake

\begin{tabular}{|c|c|c|c|c|c|}
\hline \multirow[b]{2}{*}{ Item } & \multicolumn{2}{|c|}{ Physical form of starter } & \multirow[b]{2}{*}{$\mathrm{SE}$} & \multicolumn{2}{|c|}{$P$-value ${ }^{1}$} \\
\hline & Multiparticle & Pellet & & S & $\mathrm{S} \times \mathrm{T}$ \\
\hline Initial BW, kg & 41.49 & 42.80 & 0.960 & 0.33 & - \\
\hline BW at preweaning time, $\mathrm{kg}^{2}$ & 56.63 & 57.01 & 0.957 & 0.74 & - \\
\hline Final BW, kg & 83.51 & 82.66 & 0.881 & 0.38 & - \\
\hline Initial age, $\mathrm{d}$ & 10.3 & 12.0 & 0.68 & 0.07 & - \\
\hline Preweaning age, $d$ & 49.2 & 49.2 & 0.29 & 0.98 & - \\
\hline Final age, d & 64.4 & 64.1 & 0.41 & 0.64 & - \\
\hline Total starter intake, $\mathrm{kg}$ of DM & 51.1 & 45.0 & 1.103 & $<0.01$ & $<0.01$ \\
\hline To preweaning, $\mathrm{kg}$ of $\mathrm{DM}$ & 9.57 & 9.92 & 0.953 & 0.99 & - \\
\hline Preweaning to final, $\mathrm{kg}$ of $\mathrm{DM}$ & 41.5 & 35.1 & 1.507 & $<0.01$ & - \\
\hline Total milk replacer intake, $\mathrm{kg}$ of DM & 21.73 & 21.30 & 0.550 & 0.56 & - \\
\hline $\mathrm{ADG}, \mathrm{g} / \mathrm{d}$ & 780.0 & 766.8 & 16.56 & 0.55 & 0.21 \\
\hline To preweaning, g/d & 377.4 & 390.7 & 21.60 & 0.87 & - \\
\hline Preweaning to final, g/d & $1,790.7$ & $1,742.8$ & 54.32 & 0.12 & - \\
\hline Feed conversion efficiency, $\%$ & 58.6 & 61.3 & 0.850 & 0.03 & 0.02 \\
\hline To preweaning, \% & 52.11 & 50.87 & 2.086 & 0.56 & - \\
\hline Preweaning to final, $\%$ & 63.19 & 69.02 & 1.676 & $<0.01$ & - \\
\hline
\end{tabular}

${ }^{1} \mathrm{~S}=$ effect of starter physical form; $\mathrm{S} \times \mathrm{T}=$ interaction between physical form of the starter and time.

${ }^{2}$ Preweaning is defined as the period from the beginning of the study until the time (49 $\mathrm{d}$ of age) that milk replacer offer was reduced to $2 \mathrm{~L} / \mathrm{d}$.

consuming a pelleted starter is greater than that of those consuming a multiparticle starter, and thus feeding a pelleted starter seems more economically advantageous than offering a multiparticle starter.

\section{ACKNOWLEDGMENTS}

This research was made possible in part thanks to the financial support from the Spanish Ministry of Industry, Tourism, and Trade through the CDTI (Centro para el Desarrollo Tecnológico Industrial) project number 050377.

\section{REFERENCES}

AOAC. 1990. Official Methods of Analysis. 15th ed. Association of Official Analytical Chemists, Arlington, VA.

Carnoy 2.1 for MacOSX. 2001. Laboratory of Plant Systematics, Katholieke Universiteit Leuven, Belgium.
Coverdale, J. A., H. D. Tyler, J. D. Quigley, and J. A. Brumm. 2004. Effect of various levels of forage and form of diet on rumen development and growth in calves. J. Dairy Sci. 87:2554-2562.

Franklin, S. T., D. M. Amaral-Phillips, J. A. Jackson, and A. A. Campbell. 2003. Health and performance of Holstein calves that suckled or were hand-fed colostrum and were fed one of three physical forms of starter. J. Dairy Sci. 86:2145-2153.

Kertz, A. F., B. A. Barton, and L. F. Reutzel. 1998. Relative efficiencies of wither height and body weight increase from birth until first calving in Holstein cattle. J. Dairy Sci. 81:1479-1482.

NRC. 2001. Nutrient Requirements of Dairy Cattle. 7th rev. ed. Natl. Acad. Sci., Washington, DC.

Owen, F. G., and L. L. Larson. 1986. Effect of probiocin and starter preparation on calf performance. Coop. Ext. Serv. Univ. Nebr. 1986:36-37.

Van Soest, P. J., J. B. Robertson, and B. A. Lewis. 1991. Methods for dietary fiber, neutral detergent fiber, and nonstarch polysaccharides in relation to animal nutrition. J. Dairy Sci. 74:35833597.

Warner, R. G. 1991. Nutritional factors affecting the development of a functional ruminant: A historical perspective. Pages 1-12 in Proc. Cornell Nutr. Conf., Cornell University, Ithaca, NY.

Warner, R. G., J. C. Proter, and T. S. Slack. 1973. Calf starter formulation for neonatal calves fed no hay. Pages 116-122 in Proc. Cornell Nutr. Cornell University, Ithaca, NY. 